\title{
Metabolic engineering for high yielding L(-)-carnitine production in Escherichia coli
}

\author{
Paula Arense ${ }^{1}$, Vicente Bernal ${ }^{1,4}$, Daniël Charlier ${ }^{2}$, José Luis Iborra ${ }^{1}$, Maria Remedios Foulquié-Moreno ${ }^{2,3^{*}}$ \\ and Manuel Cánovas ${ }^{1 *}$
}

\begin{abstract}
Background: L(-)-carnitine production has been widely studied because of its beneficial properties on various diseases and dysfunctions. Enterobacteria possess a specific biotransformation pathway which can be used for the enantioselective production of L(-)-carnitine. Although bioprocesses catalyzed by enzymes or whole cells can overcome the lack of enantioselectivity of chemical methods, current processes for $\mathrm{L}(-)$-carnitine production still have severe disadvantages, such as the low yields, side reactions and the need of high catalyst concentrations and anaerobic conditions for proper expression of the biotransformation pathway. Additionally, genetically engineered strains so far constructed for L(-)-carnitine production are based on plasmids and, therefore, suffer from segregational unstability.

Results: In this work, a stable, high yielding strain for L(-)-carnitine production from low cost substrates was constructed. A metabolic engineering strategy was implemented in a multiple mutant for use in both growing and resting cells systems. The effect of mutations on gene expression and metabolism was analyzed to characterize the productivity constraints of the wild type and the overproducer strains. Precise deletion of genes which encode proteins of central and carnitine metabolisms were performed. Specifically, flux through the TCA cycle was increased by deletion of acek (which encodes a bifunctional kinase/phosphatase which inhibits isocitrate dehydrogenase activity) and the synthesis of the by-product $\gamma$-butyrobetaine was prevented by deletion of caiA (which encodes a crotonobetainyl-CoA reductase). Both mutations led to improve the L(-)-carnitine production by 20 and $42 \%$, respectively. Moreover, the highly regulated promoter of the cai operon was substituted by a constitutive artificial promoter increasing the biotransformation rate, even under aerobic conditions. Resting cells of the BW $\Delta$ aceK $\Delta$ caiA p37cai strain produced $59.6 \mathrm{mmol}^{-1} \cdot \mathrm{h}^{-1}$ of $\mathrm{L}(-)$-carnitine, doubling the productivity of the wild type strain. In addition, almost total conversion was attained in less than two hours without concomitant production of the side product $\gamma$-butyrobetaine.

Conclusions: L(-)-carnitine production has been enhanced by strain engineering. Metabolic engineering strategies herein implemented allowed obtaining a robust and high yielding E. coli strain. The new overproducer strain attained almost complete conversion of crotonobetaine into L(-)-carnitine with growing and resting cells, and even under aerobic conditions, overcoming the main environmental restriction to carnitine metabolism expression. So far, this is the best performing L(-)-carnitine production E. coli strain described.
\end{abstract}

Keywords: Biotransformation, Carnitine, Strain engineering, Artificial promoter, Knock-in, Knock-out, Crotonobetaine

\footnotetext{
* Correspondence: Maria.Foulquie@mmbio.vib-kuleuven.be; mcanovas@um.es

${ }^{2}$ Research group of Microbiology, Vrije Universiteit Brussel, Pleinlaan 2,

B-1050, Brussels, Belgium

${ }^{1}$ Department of Biochemistry and Molecular Biology B and Immunology,

Faculty of Chemistry, University of Murcia, Campus de Espinardo. Regional

Campus of International Excellence "Campus Mare Nostrum", P.O. Box 4021,

Murcia E-30100, Spain

Full list of author information is available at the end of the article
} 


\section{Background}

Worldwide, the demand of L(-)-carnitine [R(-)-3-hydroxy-4-trimethylaminobutyrate] is increasing due to its multiple applications as pharmaceutical and nutraceutical product, hence the need of developing more efficient production methods. Chemical synthesis yields a racemic mixture of D,L-carnitine, which cannot be administered to patients [1-4]. The natural enantioselectivity of microbial and enzymatic biotransformations offers an advantage over classical chemical synthesis. Several biological processes have been developed for the production of L(-)-carnitine from non-chiral precursors [5-12] especially using strains belonging to the genera Escherichia and Proteus. At the industrial level, Lonza belongs a proprietary strain of a non-disclosed genus branching between Agrobacterium and Rhizobium and close to Rhizobium meliloti [13].

Crotonobetaine (dehydrated D,L-carnitine) and $\mathrm{D}(+)$-carnitine are by-products from the chemical L(-)-carnitine production process (Figure 1A), which can be transformed into L(-)-carnitine. This enantioselective biotransformation has the potential to enhance the overall economic and environmental viability of the chemical synthesis process. In this respect, the $\mathrm{L}(-)$-carnitine metabolism in $E$. coli has been widely studied and characterized [5,6,14-16] because of its role in anaerobic respiration, and stress survival, especially in osmoprotection [17-19]. E. coli is able to transform crotonobetaine into L(-)-carnitine through a series of sequential steps. Substrates and products are transported by a specific membrane antiporter (CaiT) [20]. All biochemical steps occur at the level of coenzyme A thioesters: activation of betaines involves an ATP-dependent CoAligase (CaiC), and a crotonobetainyl-CoA:carnitine CoAtransferase (CaiB) which inexpensively exchanges the CoA moiety between betaines [16,21-23]. The enantioselective hydration is catalyzed by a crotonobetainyl-CoA hydratase (CaiD) $[16,22]$. As a side reaction, crotonobetaine can be reduced to $\gamma$-butyrobetaine by means of a crotonobetainylCoA reductase (CaiA) [16,24], a respiration process which is inhibited by electron acceptors such as oxygen or fumarate (Figure 1B). All these activities are encoded by two divergent operons: caiTABCDE, which encodes the carnitine biotransformation enzymes [25], and fix $A B C X$, which encodes putative flavoproteins involved in anaerobic carnitine respiration [26,27]. Both are expressed from a common intergenic promoter-operator region, which is tightly regulated by cAMP-CRP, FNR, and the specific transcriptional activator CaiF [28]. Expression of caiF is activated by cAMP-CRP and FNR, which regulates the expression of hundreds of genes under anaerobic conditions [29-31]. So far, carnitine production by $E$. coli is carried out in anaerobic conditions to induce the expression of the cai operon, as described in the current model of regulation $[5,6,15]$.

With the aim of developing a combined and sustainable chemical-biotechnological process for industrial production of $\mathrm{L}(-)$-carnitine, we have previously reported several strategies to enhance the biotransformation of inexpensive substrates such as crotonobetaine or D (+)-carnitine. Previous approaches focused on the use of high cell density cultures, immobilized or resting cells $[11,14,32]$. Up to $40-60 \%$ crotonobetaine conversion was obtained with the wild type, non pathogenic, E. coli O44K74 strain [32,33], and $60-70 \%$ using recombinant $E$. coli strains overexpressing either the carnitine-CoA ligase or the crotonobetainyl-CoA hydratase genes (encoded by caiC and caiD, respectively) [34,35].

The major drawbacks of previous processes are the low conversion yields and the production of the sideproduct $\gamma$-butyrobetaine. Despite high volumetric productivities, the conversion yield could be improved, since the presence of excess (non-transformed) substrates and byproducts in the biotransformation medium seriously hinders downstream processing. Moreover, the cai/fix operons are only expressed under anaerobic conditions, with a concomitantly decreased energetic efficiency, and the need to supplement the medium with fumarate to inhibit the carnitine respiration pathway. In addition, using plasmid-transformed strains in large scale cultivation
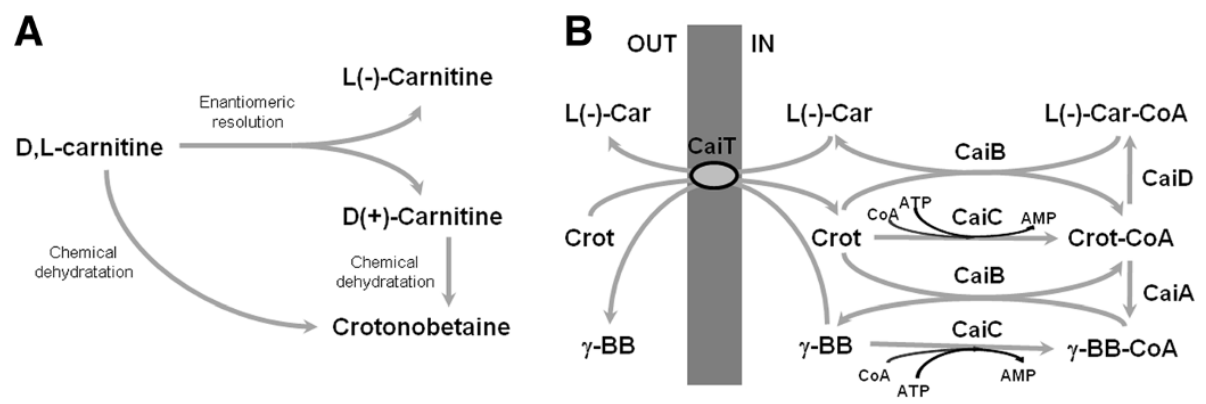

Figure 1 Synthesis of L(-)-carnitine. (A) Chemical synthesis of L(-)-carnitine and crotonobetaine. (B) Metabolism of trimethylammonium compounds in E. coli. Biotransformation of crotonobetaine into L(-)-carnitine. Abbreviations: L(-)-Car: L(-)-carnitine; Crot: crotonobetaine; $ү$-BB: Y-butyrobetaine; CaiT: L(-)-carnitine/crotonobetaine/Y-butyrobetaine protein transporter; CaiB: crotonobetainyl-CoA:L(-)-carnitine CoA-tranferase; CaiC: L(-)-carnitine, crotonobetaine or $\gamma$-butyrobetaine CoA-ligase; CaiD: crotonobetainyl-CoA hydratase. Adapted from [6]. 
presents several drawbacks such as the dependence on expensive inducers and antibiotics. Moreover, plasmids can be lost as a result of inefficient segregation between daughter cells and the high metabolic burden imposed by the maintenance of this extra genetic material $[14,36]$. Overall, all these constraints further restrict the economics of the bioprocess, preventing its implementation in an industrial scale.

This work aims at improving $\mathrm{L}(-)$-carnitine production in $E$. coli by strain engineering techniques, overcoming the major drawbacks previously exposed. All modifications were performed at the chromosomal level in order to obtain genetically stable, marker-free, high-yielding strains.

\section{Results}

\section{Strain engineering for $\mathrm{L}(-)$-carnitine production}

On the basis of previous knowledge, three strategies were designed to enhance carnitine production, dealing with either central or secondary metabolism: (i) altering the glyoxylate shunt/TCA cycle flux ratio at the isocitrate node, (ii) avoiding the reduction of crotonobetaine to $\gamma$ butyrobetaine (carnitine respiration pathway), and (iii) enhancing the expression of the $\mathrm{L}(-)$-carnitine operon structural (caiTABCDE) or regulatory genes (caiF) to relieve repression by aerobic conditions (Figure 2). All modifications were performed in E. coli BW25113, in which L (-)-carnitine productivity is in the same order of magnitude as in the well characterized $E$. coli O44K74 strain. To determine the effect on L(-)-carnitine production, the strains were cultured anaerobically in LB-CB medium.

First, as regards the modification of central metabolism, the genes encoding isocitrate lyase $($ ace $A)$ and isocitrate dehydrogenase phosphatase/kinase (aceK) were deleted. The $a c e K$ knockout strain (devoid of post-translational control of isocitrate dehydrogenase) showed a modest improvement in the production of L(-)-carnitine (20\%), while deletion of aceA (encoding the first enzyme of the glyoxylate shunt) only had a slight effect (6\%) (Figure 3 ). No further improvement was observed in the aceAK double mutant.

Second, to avoid the reduction of crotonobetainyl-CoA into $\gamma$-butyrobetainyl-CoA, the caiA gene (encoding the crotonobetainyl-CoA reductase) was deleted (Figure 1), leading to an improvement in $\mathrm{L}(-)$-carnitine production of $42 \%$ (Figure 3 ). The side-reaction was effectively suppressed since no $\gamma$-butyrobetaine was detected in the supernatant of any of the $\Delta$ caiA strains assayed (results not shown).

Furthemore, the expression of the carnitine metabolism structural (cai operon) and regulatory (caiF) genes was tuned using artificial promoters (Figure 2), eliminating the repression by aerobic conditions. When the endogenous promoters were replaced by constitutive artificial promoters, L(-)-carnitine production increased $51 \%$ and $20 \%$ in the BW p37cai and BW p8caiF strains, respectively (Figure 3 ).

Altogether, statistically significant differences in the specific L(-)-carnitine production rates were observed

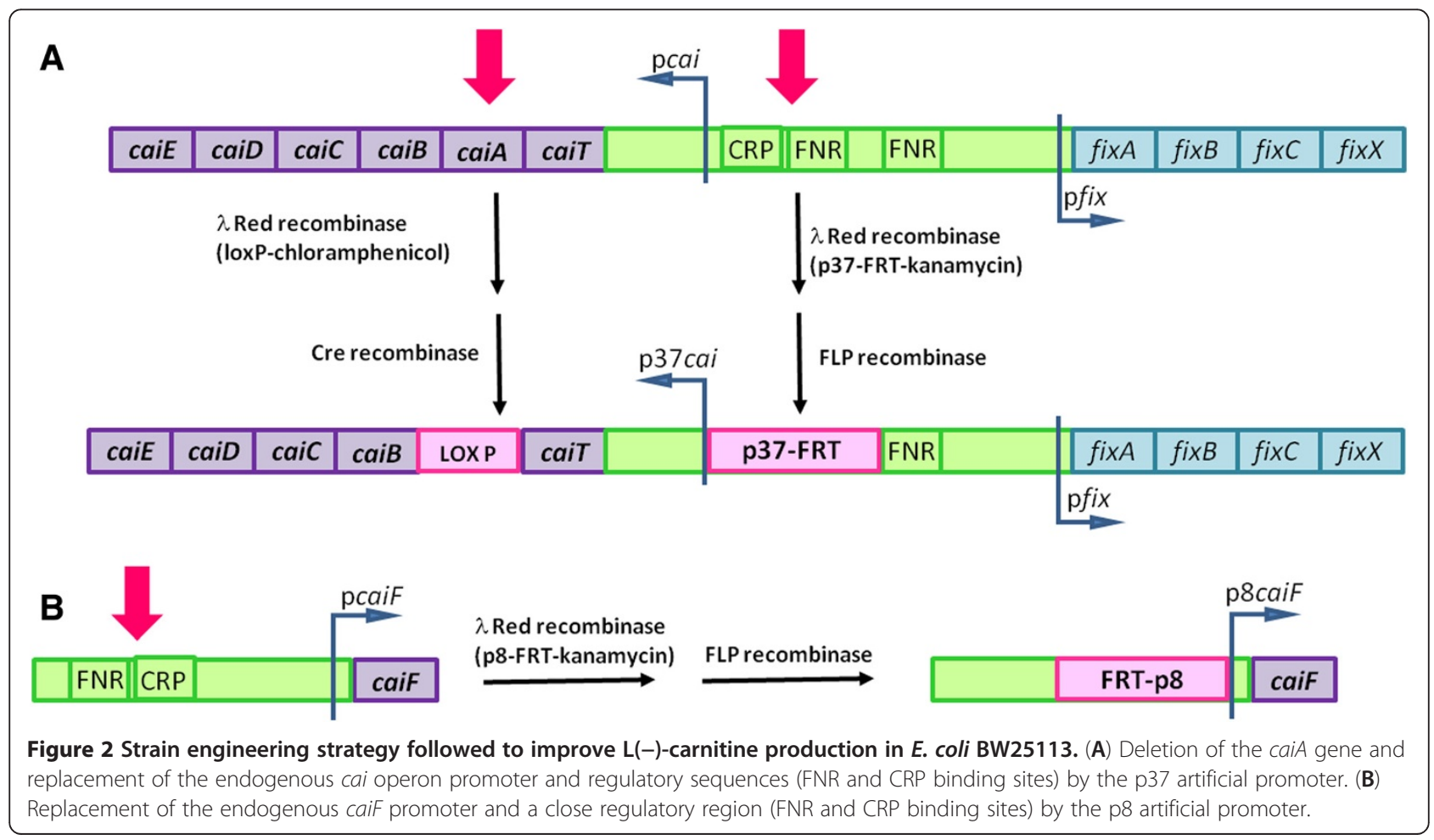




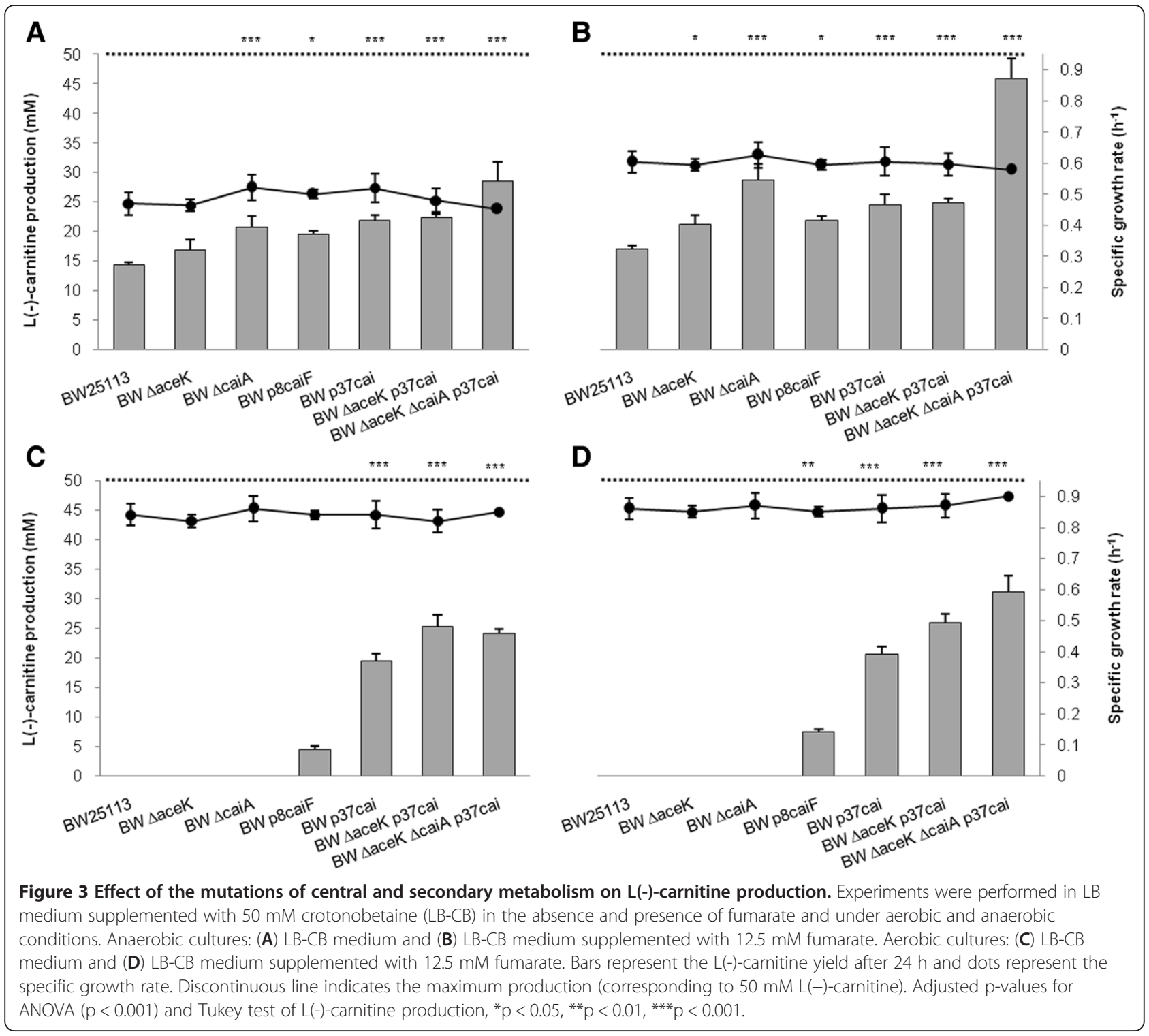

between the assayed strains. The highest $\mathrm{L}(-)$-carnitine titers were achieved by the BW p37cai and BW $\Delta$ caiA strains (21.7 and $20.7 \mathrm{mM}$ respectively) (Figure $3 \mathrm{~A}$ ).

To further study these strains with single modifications, growth and carnitine production rates were also determined in anaerobic cultures in the presence of fumarate (Figure 3B) (used as an alternative electron acceptor [24,33]). The BW $\Delta c a i A$ strain reached the highest production, $28.7 \mathrm{mM}(\mathrm{p}<0.001)$ (Figure 3B) and a similar $20-30 \%$ increase in the specific growth rate was observed for all the strains with single modifications.

To further enhance productivity, all modifications that positively affected $\mathrm{L}(-)$-carnitine production, namely, deletion of aceK and caiA and replacement of the cai promoter, were implemented in the same strain. Given its close proximity to the cai operon promoter, the caiA gene was deleted combining FRT and loxP sites in order to avoid the deletion of the contiguous caiT gene (Figure 2). As expected, the mutations did not affect growth significantly. Nevertheless, the specific L(-)-carnitine production rate was higher than in the single mutants. In order to check the effect of the mutations in the BW $\triangle a c e K \Delta c a i A \mathrm{p} 37 c a i$ strain compared to the wild type, expression of genes belonging to the cai/fix operons was analyzed by qRT-PCR. Upstream (caiT) and downstream (caiB and caiC) genes of the deleted caiA were analyzed in order to assess whether a polar effect appeared due to this deletion. As expected, the constitutive promoter increased the expression of the carnitine metabolism genes. However, caiB and caiC exhibited a lower level of expression in the $\triangle$ caiA strains (Table 1 ), indicating that caiA deletion exerts a polar effect. In 
Table 1 Relative gene expression in the engineered strains growing anaerobically on LB-CB medium supplemented with $12.5 \mathrm{mM}$ fumarate

\begin{tabular}{lcccc}
\hline Strains & cait & caiB & caic & fixA \\
\hline BW $\Delta$ caiA & $1.443 \pm 0.20$ & $0.86 \pm 0.21$ & $0.81 \pm 0.06$ & $1.32 \pm 0.19$ \\
\hline BW $\triangle$ aceK p37cai & $3.00 \pm 0.16$ & $3.08 \pm 0.24$ & $2.18 \pm 0.20-$ & $1.95 \pm 0.17$ \\
\hline BW $\Delta$ aceK $\Delta$ caiA & $3.23 \pm 0.17$ & $1.76 \pm 0.10$ & $1.47 \pm 0.12$ & $1.84 \pm 0.10$ \\
p37cai & & & &
\end{tabular}

For each gene, the transcription level of that gene in the wild type strain was used as reference to normalize the data. Relative gene expression in the wild type strain is, therefore, taken as 1 . The results are the averages of three independent measurements of each gene/condition in three independent experiments.

addition, the p37 promoter also enhanced expression of the fix operon (Table 1). This finding is not surprising, since the cai and fix operons are expressed from a common intergenic control region [29].

Furthermore, the single (BW $\triangle a c e K$ and BW $\Delta c a i A$ ), double (BW $\triangle a c e K \mathrm{p} 37 c a i$ ), and triple (BW $\triangle a c e K$ $\triangle$ caiA p37cai) mutants and the wild type strain were grown in the absence (Figure $3 \mathrm{~A}$ ) and presence of fumarate (Figure $3 \mathrm{~B}$ ) under anaerobic conditions in $\mathrm{LB}-\mathrm{CB}$ medium (Figure 3A, B). The combination of all three mutations contributed to the highest increase of specific L(-)-carnitine production rate (which doubled both in absence and presence of fumarate) and yield (reaching $70 \%$ and $92 \%$ of conversion, respectively). The highest titer obtained was $46 \mathrm{mM} \mathrm{L(-)-carnitine} \mathrm{with} \mathrm{the} \mathrm{BW}$ $\Delta$ aceK $\Delta$ caiA p37cai strain (Table 2, Figure 3B).

\section{Biotransformation under aerobic conditions}

As described before, it might be desirable to produce $\mathrm{L}$ (-)-carnitine under aerobic conditions with engineered strains that constitutively overexpressed the caiF gene and the cai operon. Tuning gene expression with artificial oxygen-independent promoters should allow reaching this goal and overcoming the limitations exhibited by the wild type strain $[37,38]$.

Indeed, the engineered strains produced L(-)-carnitine under aerobic conditions when either the endogenous promoters pcaiF or pcai were replaced by the constitutive promoters $\mathrm{p} 8$ or $\mathrm{p} 37$, respectively. As expected, the wild type, BW $\triangle a c e K$, and BW $\triangle c a i A$ strains were not able to produce L(-)-carnitine (Figure $3 \mathrm{C}, \mathrm{D})$. The presence of fumarate did not affect the maximum specific growth rate, being $0.84 \mathrm{~h}^{-1}$ for cultures without fumarate and $0.86 \mathrm{~h}^{-1}$ for cultures supplemented with fumarate, although enhanced $\mathrm{L}(-)$-carnitine production as in anaerobic cultures (Figure $3 \mathrm{C}$ and $\mathrm{D})$. The L(-)-carnitine yield was higher in the strains that harbored the p37promoter upstream the cai operon. Nevertheless, the activity of the promoter p8 was sufficient to activate the expression of carnitine metabolism and to produce $\mathrm{L}$ (-)-carnitine aerobically in the BW p8caiF strain. For p37-mutants, the biotransformation yield ranged between $40-60 \%$. In spite of the fact that the strain with the highest specific carnitine production rate was BW $\triangle a c e K$ p37cai, the strain BW $\triangle a c e K \Delta c a i A$ p37cai showed the highest L(-)-carnitine yield when reached the stationary phase in the presence of fumarate $(\mathrm{p}<$ 0.001 ), obtaining $31.2 \mathrm{mM} \mathrm{L}(-)$-carnitine. Altogether, the modifications performed allowed aerobic L(-)-carnitine production, although titers obtained were lower than under anaerobic conditions (Figure 3).

\section{Biotransformation with resting cells}

Biotransformation assays were performed with resting cells of the wild type and the BW $\Delta a c e K \Delta$ caiA p37cai strain. Carnitine production was enhanced by resting conditions in both strains. The productivities were 28.5 and $59.6 \mathrm{mmol} \mathrm{l}^{-1} \cdot \mathrm{h}^{-1}$ for the wild type and the BW $\triangle a c e K \Delta c a i A$ p37cai mutant, respectively. The mutant strain reached almost $100 \%$ conversion in less than two hours (Figure 4), which is the highest conversion ever reported for L(-)-carnitine producing E. coli strains.

\section{Effect of mutations on control points of the central metabolism}

The wild type and the BW $\Delta a c e K \Delta$ caiA p37cai mutant were selected to study the changes imposed by the genetic modifications on central metabolism. To this end, seven metabolites were analyzed: succinate, pyruvate, fumarate, lactate, acetate, ethanol, and formate. Major changes were observed on acetate, which is the main metabolic product and can be considered as readout of the energetic state, and on succinate, which is a product of the mixed acid fermentation pathway and also results from fumarate respiration (Table 2).

In anaerobic cultures supplemented with fumarate, the wild type and the mutant strain exhibited a similar behavior. The maximum concentration of succinate and acetate coincided with the end of the exponential phase, when the culture broth was completely depleted of fumarate (12.2 and $13.9 \mathrm{mM}$ succinate, and 10.2 and $16.4 \mathrm{mM}$ acetate for the wild type and the overproducer strain, respectively). Acetate decreased slightly during the stationary phase. A similar behavior was observed in the assays without fumarate. Formate was only detected in the exponential phase, increasing steeply during the first ten hours of culture, especially in the mutant strain. It was not detected in the stationary phase of cultures, which indicates the activity of formate hydrogen-lyase (Fhl) under these conditions. Changes observed in the fermentation profiles in anaerobic cultures are especially relevant, since they reflect the energy state of cells. Thus, the central metabolism of E. coli strains was focused on maintaining suitable levels of ATP and free coenzyme A, 
Table 2 Metabolic performance of wild-type (BW25113) and engineered (BW $\Delta a c e K \Delta c a i A$ p37cai) E. coli strains during L(-)-carnitine production in anaerobic and aerobic conditions

\begin{tabular}{|c|c|c|c|c|c|c|}
\hline Strain and conditions & $\mathrm{q}_{\mathrm{L}-\mathrm{car}}$ & $\mathrm{q}_{\text {suc }}$ & $-q_{\text {Fum }}$ & $q_{\text {Acet }}$ & $q_{\text {EtOH }}$ & $q_{\text {Form }}$ \\
\hline \multicolumn{7}{|l|}{ Anaerobic cultures } \\
\hline \multicolumn{7}{|l|}{$\mathrm{LB}-\mathrm{CB}$} \\
\hline BW25113 & $9.30 \pm 0.12$ & $8.43 \pm 0.22$ & - & $15.71 \pm 0.28$ & $7.23 \pm 0.20$ & $4.21 \pm 0.14$ \\
\hline BW $\Delta$ acek $\Delta$ caiA p37cai & $18.87 \pm 0.23^{* * *}$ & $7.20 \pm 0.17^{* * *}$ & - & $17.44 \pm 0.16^{* * *}$ & $2.49 \pm 0.12^{* * *}$ & $12.96 \pm 0.42^{* * *}$ \\
\hline \multicolumn{7}{|l|}{ LB-CB + fumarate (12.5 mM) } \\
\hline BW25113 & $14.23 \pm 0.15$ & $13.89 \pm 0.54$ & $17.74 \pm 0.26$ & $13.15 \pm 0.36$ & $4.8 \pm 0.32$ & N.D. \\
\hline BW $\Delta$ acek $\Delta$ caiA p37cai & $27.75 \pm 0.17^{* * *}$ & $10.31 \pm 0.32^{* * *}$ & $19.51 \pm 0.41^{* * *}$ & $16.44 \pm 0.21^{* * *}$ & $3.95 \pm 0.27^{* * *}$ & N.D. \\
\hline \multicolumn{7}{|l|}{ Aerobic cultures } \\
\hline \multicolumn{7}{|l|}{ LB-CB } \\
\hline BW25113 & - & N.D. & - & $9.49 \pm 0.23$ & $0.43 \pm 0.05$ & - \\
\hline BW $\Delta$ acek $\Delta$ caiA p37cai & $6.15 \pm 0.10^{* * *}$ & N.D. & - & $7.79 \pm 0.12^{* * *}$ & $1.17 \pm 0.08^{*}$ & - \\
\hline \multicolumn{7}{|l|}{ LB-CB + fumarate (12.5 mM) } \\
\hline BW25113 & - & $3.32 \pm 0.11$ & $9.42 \pm 0.24$ & $5.74 \pm 0.10$ & $0.15 \pm 0.02$ & - \\
\hline BW $\Delta$ acek $\Delta$ caiA p37cai & $7.02 \pm 0.07^{* * * *}$ & $2.95 \pm 0.08$ & $6.82 \pm 0.17^{* * *}$ & $8.31 \pm 0.14^{* * *}$ & $1.26 \pm 0.06^{* * *}$ & - \\
\hline
\end{tabular}

Specific production/consumption rates of $\mathrm{L}(-)$-carnitine and the main extracellular metabolites were calculated during the early exponential phase of cultures. All rates are expressed in mmol g $\mathrm{m}^{-1}$. Pairwise statistical comparison of the parameters assessed for both strains was perfomed with ANOVA and Tukey tests. Adjusted $\mathrm{p}$-values are indicated as follows: ${ }^{*} \mathrm{p}<0.05$, ${ }^{* *} \mathrm{p}<0.01$, ${ }^{* *} \mathrm{p}<0.001$.

which are a limiting factor in the biotransformation process.

The strains grown aerobically showed similar patterns in the metabolite profiles, although differences were found in the levels reached in the stationary phase. At the beginning of the stationary phase, succinate and acetate achieved their maximum concentration $(8.3$ and $18.1 \mathrm{mM}$, respectively, for the mutant; 7.4 and $12.5 \mathrm{mM}$, respectively, for the wild type strain), emphasizing the importance of the acetate metabolism. Moreover, acetate decreased drastically in the later stationary phase, indicating the activity of the acetate scavenging systems
[39-41]. Similar acetate profiles were observed in cultures without fumarate. Under aerobic conditions, acetate overflow highlights the need of maintaining the acetyl-CoA/CoASH ratio to meet cellular demands.

\section{Discussion}

This work demonstrates the successful construction of E. coli strains engineered for high yielding production of L(-)-carnitine from crotonobetaine. Biotransformation yields ranging from 40 to $95 \%$ were obtained in the growing cells system, while conversion was almost complete using resting cells. The strain optimization

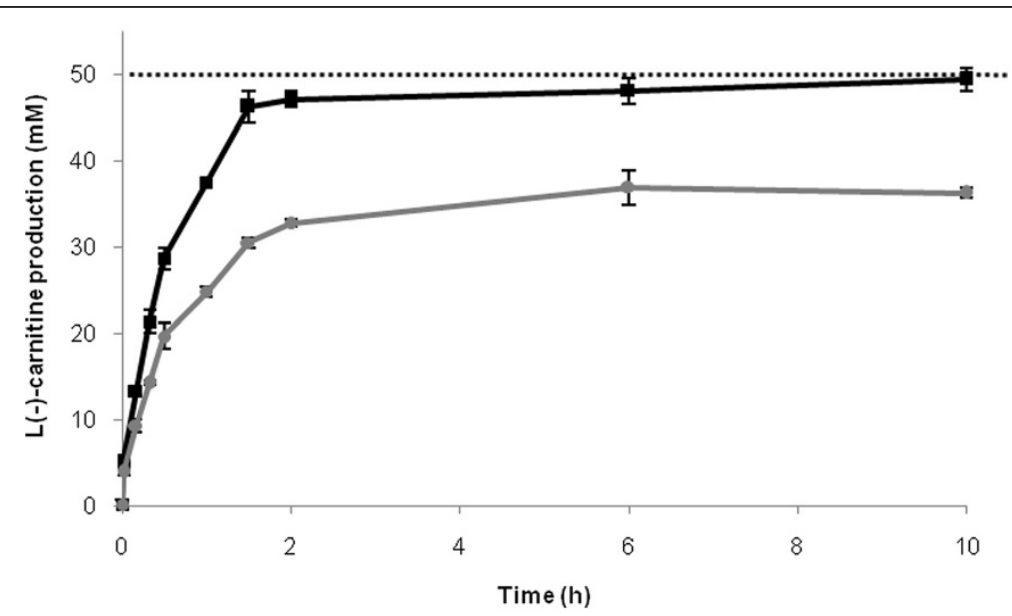

Figure $4 \mathrm{~L}(-)$-carnitine production by resting cells. The performance of the BW25113 (wild type) (grey) and BW25113 $\Delta$ aceK $\Delta$ caiA p37cai (black) strains is compared. Discontinuous line indicates the maximum production (corresponding to $50 \mathrm{mM} \mathrm{L(-)-carnitine).} \mathrm{Resting} \mathrm{cell}$ experiments were performed in phosphate buffered $50 \mathrm{mM}$ crotonobetaine, as explained in the Methods section. 
strategy presented here intended to overcome the major drawbacks previously identified. The modifications introduced had a cumulative effect on strain performance, improving yield and productivity without affecting growth or physiology of the bacteria. In fact, the best results were obtained with the BW $\triangle a c e K \Delta$ caiA p37cai strain which had a growth profile similar to that of the wild type strain.

Previous works demonstrated that the optimization of the biotransformation process depends on primary and secondary metabolisms and its regulation [15,18,34,42,43]. Knockout mutants on the glyoxylate shunt genes aceK and ace $A$ showed a modest increase in carnitine production [34], underlining the importance of the TCA cycle flux. However, its impact on the productivity was small when compared to the overexpression of enzymes performing the biotransformation such as CaiC and $\mathrm{CaiB}$ $[23,34,35]$ or the whole cai operon (this work).

The replacement of the promoter of the caiF gene with the constitutive $\mathrm{p} 8$ promoter enhanced L(-)-carnitine production under aerobic and anaerobic conditions. CaiF is a specific transcriptional activator of carnitine metabolism, binding to two 11-bp inverted repeat halfsites separated by $13 \mathrm{bp}$ in the intergenic region of the two operons caiTABCDE and fix $A B C X$. The expression of $c a i F$ is activated by cAMP-CRP and FNR which prevents the expression of the trimethylammonium compounds metabolism in the presence of oxygen and/or glucose [28-30]. Transcriptional repression was overcome after promoter replacement in BW p8caiF, although this was not sufficient for optimal performance, especially under aerobic conditions. Various reasons can respond for this observed effect. For instance, although the FNR binding site located at -55.5 bp in the caiF promoter was replaced, the presence of further putative half sites for FNR binding is known, which role on caiF expression is not known. In addition, the cai promoter is also regulated by FNR (two sites at -90.5 and $-41.5 \mathrm{bp}$, respectively) and ArcA (four sites located at $-101,-91,+28$, and $+50 \mathrm{bp}$ ) [44], and CaiF overexpression could not be enough for optimal expression under aerobic conditions.

The most remarkable improvement was obtained by tuning the expression of the cai operon and deleting caiA gene. Replacement of the endogenous promoter by the constitutive promoter p37 enhanced L(-)-carnitine production under anaerobic and aerobic conditions, relieving the cai operon from the regulatory effects of oxygen and CaiF. Importantly, the formation of the sideproduct $\gamma$-butyrobetaine was effectively avoided by deleting caiA, redirecting crotonobetaine towards $\mathrm{L}$ (-)-carnitine production [16,24]. This deletion led to a $25-60 \%$ enhancement in $\mathrm{L}(-)$-carnitine production in the BW $\Delta$ caiA strain and a $30-87 \%$ enhancement in the BW $\triangle a c e K \Delta$ caiA p37cai strain. This is the best performing strain obtained, achieving over 95\% conversion of the substrate in a growing system, and almost $100 \%$ of conversion in a resting cells system.

When the effect of the metabolic engineering strategy on the physiology of the bacteria was assessed, several metabolic changes between the wild type and the BW $\Delta a c e K \Delta$ caiA p37cai strain were observed. Under anaerobic biotransformation conditions, energy producing processes such as fumarate respiration $[45,46]$ and acetate and formate production [40,41,47-50] were enhanced in the mutant. This supports the high dependence of $\mathrm{L}$ (-)-carnitine production on the energetic state of cells [15]. Although fumarate was originally used as electron acceptor and inhibitor of the crotonobetainyl-CoA reductase activity (CaiA) [15,24,33], media supplementation with this energetic substrate enhanced growth and $\mathrm{L}(-)$-carnitine production, even in $\Delta$ caiA strains. Therefore, the reduction of fumarate is a major mechanism of ATP generation in anaerobic conditions $[15,42,45]$, similarly to aerobic oxidative phosphorylation. Increased production of acetate (the end-product of the major energy producing anaerobic pathway of $E$. coli) and formate (the end product of pyruvate-formate lyase) was not observed in aerobic cultures, in which energy is produced by respiration and acetate production occurs as a result of an overflow metabolism [39-41].

It is important to emphasize that the engineered strains created in this work were able to carry out the biotransformation under aerobic conditions, while no $\mathrm{L}$ (-)-carnitine was produced by wild type $E$. coli. The best performing strain was BW $\triangle a c e K \Delta c a i A$ p37cai with $65 \%$ of conversion, a 4-fold higher yield than that reported in previous works under aerobic conditions [33,34]. Fast growth and low biotransformation rate of the BW $\triangle a c e K$ $\triangle c a i A$ p37cai strain avoided complete conversion of crotonobetaine. This suggests that other limitations in central metabolism may occur. A plausible explanation for the observed differences between both conditions could be coenzyme A availability, as shown in previous works $[15,34,42]$.

Summarizing, we have successfully engineered $E$. coli for efficient, high-yielding L(-)-carnitine production from an inexpensive substrate (such as crotonobetaine). A stable engineered strain was obtained, which does not depend on expensive inducers (since the p37 and p8 promoters are constitutive) or antibiotics (since all modifications are chromosomal, stable and antibiotic marker-free). In addition, fast transformation was almost complete, therefore with improved downstream processing. Exploitation of this engineered strain in high-density reactors is a feasible and economically viable strategy for the implementation of $\mathrm{L}(-)$-carnitine production processes at the industrial scale. 


\section{Conclusions}

$\mathrm{L}(-)$-carnitine production in E. coli based growing and resting cells systems has been successfully improved. Multiple stable mutations introduced in a single strain enhanced production without reducing cell viability or affecting specific growth. Furthermore, the biotechnological process was improved and allowed nearly 100\% conversion reducing the time of transformation and simplifying downstream processing. Moreover, the main restriction to aerobic expression of the carnitine metabolism was eliminated. This study presents a successful strain improvement strategy by means of gene deletion and promoter replacement and contributes to get further insights into the secondary metabolism of trimethylammonium compounds in E. coli.

\section{Methods}

\section{Strains and plasmids}

The wild type strain E. coli BW25113 [lacl ${ }^{\mathrm{q}} \quad \mathrm{rrnB}_{\mathrm{T} 14}$ $\Delta l a c Z_{\mathrm{WJ16}}$ hsdR514 $\left.\triangle a r a B A D_{\mathrm{AH} 33} \quad \Delta r h a B A D_{\mathrm{LD78}}\right]$ was obtained from the Keio collection [51]. The mutant strains constructed in the present study (Table 3) were obtained as described below. The strains were stored in $50 \%$ glycerol at $-80^{\circ} \mathrm{C}$.

Standard E. coli cultures for molecular biology work were performed in Luria-Bertani broth (LB). Antibiotics (ampicillin $100 \mu \mathrm{g} \mathrm{mL}^{-1}$, kanamycin $30 \mu \mathrm{g} \mathrm{mL}{ }^{-1}$, chloramphenicol $30 \mu \mathrm{g} \mathrm{mL}^{-1}$ ) were added whenever necessary.

The plasmids pKD46 (Red helper plasmid, Ampicillin resistance), pKD3 (containing a FRT-flanked chloramphenicol resistance (cat) gene), pKD4 (containing a FRT-flanked kanamycin resistance (kan) gene), pCP20 (expressing FLP recombinase activity) [52,53], and pKDCre (expressing Cre recombinase activity) were obtained from Prof. Dr. J-P Hernalsteens (Vrije Universiteit Brussels, Belgium). The chloramphenicol resistant (cat) gene flanked by loxP sites and the priming P1 and P2 sites was cloned into pBlueScript using XbaI and BamHI restrictions sites.
All molecular biology experimentation and strain engineering performed for the completion of this work were approved by the Bioethics Committee of the University of Murcia and complies with all legal requirements.

\section{Strain engineering: gene knock-out and promoter knock- in strategies}

Standard molecular biology protocols were used [54]. Knockout mutants were constructed by successive deletion of specifically targeted genes or regulatory regions using the method of Datsenko and Wanner [53]. Targeted sequences were PCR-amplified using specifically designed primers (see Additional file 1: Table S1) and transformed into pKD46-carrying cells. Mutants were selected for either kanamycin or chloramphenicol resistance. The pCP20-encoding FLP recombinase protein or $\mathrm{pKD}$-Cre-encoding Cre recombinase protein was used to excise the antibiotic-resistance cassette. For the mutation of both cai/fix and caiF promoters, the promoter knock-in method was used [37]. The specific strategy consisted in the replacement of the respective endogenous promoter sequences by synthetic promoters. Knock-in mutants were constructed from these deletion strains. Two constitutive promoters with different strength were tested for the tuning strategy: p37 (strong) and p8 (weak). These synthetic promoters have been previously described [38] (Figure 2). The mutant strains constructed are listed in Table 3. All constructions were checked by $\mathrm{PCR}$ and DNA sequencing.

\section{Culture conditions}

For the biotransformation of L(-)-carnitine from crotonobetaine, a pre-culture was grown at $37^{\circ} \mathrm{C}$ aerobically using $\mathrm{LB}$ medium, $\mathrm{pH}$ adjusted to 7.5 with $\mathrm{KOH}$ prior to autoclaving (LB). The cultures were inoculated with 3\% $(\mathrm{v} / \mathrm{v})$ of an overnight grown pre-culture. Cultures were grown under both aerobic and anaerobic conditions at $37^{\circ} \mathrm{C}$ in $\mathrm{LB}$ medium supplemented with $50 \mathrm{mM}$ crotonobetaine as substrate (LB-CB). In some cultures,

Table 3 List of bacterial strains used in this work

\begin{tabular}{|c|c|c|c|}
\hline Strain & References & Genotype & Short name \\
\hline E. coli BW25113 & Keio collection, Baba et al. [51] & 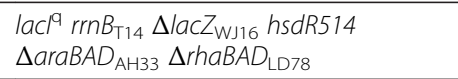 & BW25113 \\
\hline E. coli BW25113 $\Delta$ aceK & This work & [BW25113] $\Delta$ aceK & BW $\Delta$ acek \\
\hline E. coli BW25113 $\triangle a c e A$ & This work & [BW25113] $\Delta$ aceA & BW $\Delta a c e A$ \\
\hline E. coli BW25113 $\triangle$ aceAK & This work & [BW25113] $\Delta$ aceAK & BW $\triangle a c e A K$ \\
\hline E. coli BW25113 $\Delta$ caiA & This work & [BW25113] $\Delta$ caiA & BW $\Delta$ caiA \\
\hline E. coli BW25113 $\Delta$ pcaif caiF-p8 & This work & [BW25113] $\Delta$ pcaiF::caiF-p8 & BW p8caif \\
\hline E. coli BW25113 $\Delta$ pcai cai-p37 & This work & [BW25113] $\Delta$ pcai::cai-p37 & BW p37cai \\
\hline E. coli BW25113 $\Delta$ aceK $\Delta$ pcai cai-p37 & This work & [BW25113] $\Delta$ aceK $\Delta$ pcai::cai-p37 & BW $\Delta a c e k$ p37cai \\
\hline E. coli BW25113 $\Delta$ aceK $\Delta$ caiA $\Delta$ pcai cai-p37 & This work & [BW25113] $\Delta$ aceK $\Delta$ caiA $\Delta$ pcai::cai-p37 & BW $\Delta$ acek $\Delta c a i A$ p37cai \\
\hline
\end{tabular}


fumarate $12.5 \mathrm{mM}$ was added acting as electron acceptor and as inhibitor of the reaction catalyzed by the crotonobetainyl-CoA reductase (CaiA). Batch anaerobic assays were performed in $100 \mathrm{~mL}$ vessels with $60 \mathrm{~mL}$ working volume under nitrogen atmosphere and magnetic stirring. Aerobic assays were performed in $250 \mathrm{~mL}$ erlenmeyer flasks with $50 \mathrm{~mL}$ working volume in a rotary shaker $(150 \mathrm{rpm})$. The experiments were performed in triplicate.

\section{Resting cells}

For the resting cell assays, anaerobic cultures in LB medium with $5 \mathrm{mM}$ of crotonobetaine, used as inducer of cai operon, were harvested at the end of the exponential growth phase, centrifuged at 16,000xg for $10 \mathrm{~min}$, and washed twice with $67 \mathrm{mM}$ potassium phosphate buffer, $\mathrm{pH}$ 7.5. Cells were resuspended in $50 \mathrm{mM}$ potassium phosphate buffer, $\mathrm{pH} 7.5$ with $50 \mathrm{mM}$ crotonobetaine and incubated at $37^{\circ} \mathrm{C}$ in erlenmeyer flasks in a rotary shaker (150 rpm). All experiments were performed at least in triplicate and under sterile conditions.

\section{Analytical procedures}

Cell growth was followed by optical density (OD) at $600 \mathrm{~nm}$ with a spectrophotometer (Novaspec II; Pharmacia-LKB, Sweden) and converted to dry cell weight (DWC). For L(-)-carnitine and extracellular metabolite analysis, cell-free supernatant was obtained by centrifugation at $19,000 \mathrm{xg}$ for $10 \mathrm{~min}$. L(-)-carnitine concentration was determined with an enzymatic assay [32].

$\gamma$-Butyrobetaine was determined by HPLC [32] with a Spherisorb- $\mathrm{NH}_{2}$ column $(3 \mu \mathrm{m}, 4.6 \times 150 \mathrm{~mm})$ supplied by Waters (Barcelona, Spain). The isocratic mobile phase was acetonitrile $/ \mathrm{H}_{3} \mathrm{PO}_{4} 0.005 \mathrm{M} \mathrm{pH} 5.5(65 / 35)$ at a flow rate of $1 \mathrm{~mL} \mathrm{~min}{ }^{-1}$. For the analysis of fermentation products (acetate, ethanol, formate, fumarate, pyruvate, and succinate), a cation exchange Aminex HPX-87H column supplied by BioRad Labs (Hercules, CA) was used. The isocratic mobile phase was $5 \mathrm{mM} \mathrm{H}_{2} \mathrm{SO}_{4}$ at a flow rate of $0.5 \mathrm{~mL} \mathrm{~min}{ }^{-1}$. A HPLC system from Shimadzu (Kyoto, Japan) was used. The effluent was monitored using diode array and refractive index detectors (Shimadzu, Kyoto, Japan).

\section{RNA isolation and quantitative PCR}

RNA was isolated at mid-exponential phase, when $\mathrm{L}$ (-)-carnitine production rate was maximum. The cultures were pelleted by centrifugation at $15,000 \times g$ at $4^{\circ} \mathrm{C}$ for $30 \mathrm{~s}$. Total RNA was isolated by Qiagen Rneasy ${ }^{\circ} \mathrm{Mini}$ Kit (QIAGEN Ibérica, Madrid, Spain). Additionally, DNaseI digestion of the isolated RNA was performed using the RNase-free DNase Set (QIAGEN Ibérica, Madrid, Spain) to avoid DNA interferences during PCR steps. RNA quality and quantity were evaluated by microfluidic capillary electrophoresis on an Agilent 2100 Bioanalyzer (Agilent Technologies, PaloAlto, CA) using Agilent RNA 6000 Pico kit. The primers used in this work were designed using the PrimerExpress ${ }^{\oplus}$ Software v3.0 (Applied Biosystems, FosterCity, CA) and ordered from Sigma-Aldrich Co (St. Louis, USA) (see Additional file 1: Table S1). The dnaA (encoding the multifunctional initiator of chromosome replication and transcriptional regulator) and polA genes (encoding the DNA polymerase I) were used as HKG. Quantitative PCR was performed in a 7300 Real-Time PCR System (Applied Biosystems,Foster City, CA) using PowerSYBR ${ }^{\circ}$ Green PCR Master Mix (Applied Biosystems, Foster City, CA). Samples were run in triplicate. Raw data were transformed into threshold cycle $(\mathrm{Ct})$ values. Relative gene expression was calculated by the comparative $\mathrm{Ct}$ method $(\Delta \Delta \mathrm{Ct})$. Experiments were performed in triplicate.

\section{Statistical analysis of data}

The statistical analyses were carried out using $\mathrm{R}$ (version 2.15.1). A one-way ANOVA was applied to determine the differences among different conditions and strains. A Tukey test was also carried out to ascertain the significant differences between data pairs. The threshold pvalue chosen for statistical significance was $\mathrm{p}<0.05$.

\section{Additional file}

\section{Additional file 1: Table S1. Primers used for real time PCR. The primers used in this work were designed using the Primer Express ${ }^{\circledR}$ Software v3.0 (Applied Biosystems, Foster City, CA) and ordered from Sigma-Aldrich (Sigma-Aldrich Co., St. Louis, USA). The dnaA and polA genes (encoding the multifunctional initiator of chromosomal replication and transcriptional regulator and DNA polymerase I, respectively) were used as internal control for relative quantification.}

\section{Competing interests}

The authors have filed a Spanish Patent Application (P201230867), based on part of the results here presented.

\section{Authors' contributions}

PA carried out the experimental assays and genetic modifications. PA and VB designed the study, analyzed the data and drafted the manuscript. JLI and DC drafted the manuscript. MRFM carried out the design of primers, participated in the genetic modifications and helped to draft the manuscript. MC conceived the study and drafted the manuscript. All authors read and approved the final version of the manuscript.

\section{Acknowledgements}

P. Arense and V. Bernal acknowledge a PhD fellowship (Fundación Séneca, CARM, Spain) and a postdoctoral contract (Universidad de Murcia, Spain), respectively. This work was funded by FEDER funds by the projects: 08660/ PI/08 (Fundación Séneca, CARM, Spain) and BIO2008-04500-C02-01 and BIO2011-29233-C02-01 (MICINN, Spain).

\section{Author details}

${ }^{1}$ Department of Biochemistry and Molecular Biology B and Immunology, Faculty of Chemistry, University of Murcia, Campus de Espinardo. Regional Campus of International Excellence "Campus Mare Nostrum", P.O. Box 4021, Murcia E-30100, Spain. ${ }^{2}$ Research group of Microbiology, Vrije Universiteit Brussel, Pleinlaan 2, B-1050, Brussels, Belgium. ${ }^{3}$ Laboratory of Molecular Cell 
Biology, KU Leuven. VIB (Flanders Institute of Biotechnology), Kasteelpark Arenberg, 31; bus 2438, Heverlee 3001, Belgium. ${ }^{4}$ Current address: Grupo de Bioenergía. Dirección de Tecnología, Centro de Tecnología de REPSOL, Carretera A-5, Km 18, Móstoles-Madrid 28935, Spain.

Received: 27 December 2012 Accepted: 7 May 2013

Published: 29 May 2013

\section{References}

1. Mescka C, Moraes T, Rosa A, Mazzola P, Piccoli B, Jacques C, Dalazen G, Coelho J, Cortes M, Terra M, Regla Vargas C, Dutra-Filho CS: In vivo neuroprotective effect of $\mathrm{L}$-carnitine against oxidative stress in maple syrup urine disease. Metab Brain Dis 2011, 26:21-28.

2. Bieber LL: Carnitine. Annu Rev Biochem 1988, 57:261-283.

3. Bremer J: Carnitine in intermediary metabolism. The biosynthesis of palmitylcarnitine by cell subfractions. J Biol Chem 1963, 238:2774-2779.

4. Fritz IB, Yue $\mathrm{KT}$ : Long-chain carnitine acyltransferase and the role of acylcarnitine derivatives in the catalytic increase of fatty acid oxidation induced by carnitine. J Lipid Res 1963, 4:279-288.

5. Kleber HP: Bacterial carnitine metabolism. FEMS Microbiol Lett 1997, 147:1-9.

6. Bernal V, Sevilla A, Cánovas M, Iborra JL: Production of L-carnitine by secondary metabolism of bacteria. Microb Cell Fact 2007, 6:31.

7. Elssner T, Preusser A, Wagner U, Kleber HP: Metabolism of L(-)-carnitine by Enterobacteriaceae under aerobic conditions. FEMS Microbiol Lett 1999, 174:295-301.

8. Tian J, Wang Q, Zhang Z: A novel strategy to improve the bioconversion of I-carnitine from crotonobetaine. Eur Food Res Technol 2009, 229:721-724.

9. Giuliano M, Schiraldi C, Maresca C, Esposito V, De Rosa M: Immobilized Proteus mirabilis in poly(vinyl alcohol) cryogels for I(-)-carnitine production. Enzym Microb Technol 2003, 32:507-512.

10. Jung H, Jung K, Kleber HP: Synthesis of L-carnitine by microorganisms and isolated enzymes. Adv Biochem Eng Biotechnol 1993, 50:21-44.

11. Cánovas M, Maiquez J, De Diego T, Buendía B, Espinosa G, Iborra JL, Canovas M, Buendia B: Membrane cell retention systems for continuous production of L-carnitine using Proteus sp. J Membr Sci 2003, 214:101-111.

12. Engemann C, Elssner T, Kleber HP: Biotransformation of crotonobetaine to L(-)-carnitine in Proteus sp. Arch Microbiol 2001, 175:353-359.

13. Kulla HG: Enzymatic hydroxylations in industrial applications. CHIMIA Int J Chem 1991, 45:81-85.

14. Cánovas M, Bernal V, González M, Kleber HP, Iborra JL: Factors affecting the biotransformation of trimethylammonium compounds into I-carnitine by Escherichia coli. Biochem Eng J 2005, 26:145-154.

15. Cánovas M, Bernal V, Torroglosa T, Ramirez $\mathrm{JL}$, Iborra JL: Link between primary and secondary metabolism in the biotransformation of trimethylammonium compounds by Escherichia coli. Biotechnol Bioeng 2003, 84:686-699.

16. Engemann C, Elssner T, Pfeifer S, Krumbholz C, Maier T, Kleber H-P: Identification and functional characterisation of genes and corresponding enzymes involved in carnitine metabolism of Proteus $\mathrm{sp}$. Arch Microbiol 2005, 183:176-189.

17. Arense P, Bernal V, Iborra JL, Cánovas M: Metabolic adaptation of Escherichia coli to long-term exposure to salt stress. Process Biochem 2010, 45:1459-1467.

18. Cánovas M, Bernal V, Sevilla A, Iborra JL: Salt stress effects on the central and carnitine metabolisms of Escherichia coli. Biotechnol Bioeng 2007 96:722-737

19. Jung $\mathrm{H}$, Jung $\mathrm{K}$, Kleber HP: L-carnitine metabolization and osmotic stress response in Escherichia coli. J Basic Microbiol 1990, 30:409-413.

20. Jung H, Buchholz M, Clausen J, Nietschke M, Revermann A, Schmid R, Jung K: CaiT of Escherichia coli, a new transporter catalyzing L-carnitine /gamma -butyrobetaine exchange. J Biol Chem 2002, 277:39251-39258.

21. Elssner T, Hennig L, Frauendorf H, Haferburg D, Kleber HP: Isolation, identification, and synthesis of gamma-butyrobetainyl-CoA and crotonobetainyl-CoA, compounds involved in carnitine metabolism of $\mathrm{E}$. coli. Biochemistry 2000, 39:10761-10769.

22. Elssner T, Engemann C, Baumgart K, Kleber HP: Involvement of coenzyme A esters and two new enzymes, an enoyl-CoA hydratase and a CoAtransferase, in the hydration of crotonobetaine to L-carnitine by Escherichia coli. Biochemistry 2001, 40:11140-11148.
23. Bernal V, Arense P, Blatz V, Mandrand-Berthelot MA, Cánovas M, Iborra JL: Role of betaine:CoA ligase (CaiC) in the activation of betaines and the transfer of coenzyme A in Escherichia coli. J App/ Microbiol 2008, 105:42-50.

24. Preusser A, Wagner U, Elssner T, Kleber HP: Crotonobetaine reductase from Escherichia coli consists of two proteins. Biochim Biophys Acta 1999, 1431:166-178

25. Eichler K, Bourgis F, Buchet A, Kleber HP, Mandrand-Berthelot MA: Molecular characterization of the cai operon necessary for carnitine metabolism in Escherichia coli. Mol Microbiol 1994, 13:775-786.

26. Eichler K, Buchet A, Bourgis F, Kleber HP, Mandrand-Berthelot MA: The fix Escherichia coli region contains four genes related to carnitine metabolism. J Basic Microbiol 1995, 35:217-227.

27. Walt $A$, Kahn ML: The fixA and fixB genes are necessary for anaerobic carnitine reduction in Escherichia coli. J Bacteriol 2002, 184:4044-4047.

28. Eichler $\mathrm{K}$, Buchet A, Lemke R, Kleber HP, Mandrand-Berthelot MA: Identification and characterization of the caiF gene encoding a potential transcriptional activator of carnitine metabolism in Escherichia coli. J Bacteriol 1996, 178:1248-1257.

29. Buchet A, Eichler K, Mandrand-Berthelot MA: Regulation of the carnitine pathway in Escherichia coli: investigation of the cai-fix divergent promoter region. J Bacteriol 1998, 180:2599-2608.

30. Buchet A, Nasser W, Eichler K, Mandrand-Berthelot MA: Positive coregulation of the Escherichia coli carnitine pathway cai and fix operons by CRP and the CaiF activator. Mol Microbiol 1999, 34:562-575.

31. Shalel-Levanon S, San KY, Bennett GN: Effect of ArCA and FNR on the expression of genes related to the oxygen regulation and glycolysis pathway in Escherichia coli under growth conditions. Biotechnol Bioeng 2005, 92:147-159.

32. Obon JM, Maiquez JR, Canovas M, Kleber HP, Iborra JL, Obón JM, Cánovas M: High-density Escherichia coli cultures for continuous L(-)-carnitine production. Appl Microbiol Biotechnol 1999, 51:760-764.

33. Castellar MR, Cánovas M, Kleber HP, Iborra JL: Biotransformation of $D$ (+)-carnitine into L(-)-carnitine by resting cells of Escherichia coli $\mathrm{O} 44$ K74. J Appl Microbiol 1998, 85:883-890.

34. Bernal V, Masdemont B, Arense P, Canovas M, Iborra JL, Cánovas M: Redirecting metabolic fluxes through cofactor engineering: Role of CoAesters pool during L(-)-carnitine production by Escherichia coli. J Biotechnol 2007, 132:110-117.

35. Castellar MR, Obón JM, Marán A, Cánovas M, Iborra JL: L(-)-carnitine production using a recombinant Escherichia coli strain. Enzym Microb Technol 2001, 28:785-791.

36. Bernal V, González-Veracruz M, Cánovas M, Iborra JL: Plasmid maintenance and physiology of a genetically engineered Escherichia coli strain during continuous L-carnitine production. Biotechnol Lett 2007, 29:1549-1556.

37. De Mey M, Maertens J, Boogmans S, Soetaert WK, Vandamme EJ, Cunin R, Foulquié-Moreno MR: Promoter knock-in: a novel rational method for the fine tuning of genes. BMC Biotechnol 2010, 10:26.

38. De Mey M, Maertens J, Lequeux GJ, Soetaert WK, Vandamme EJ Construction and model-based analysis of a promoter library for E. coli: an indispensable tool for metabolic engineering. BMC Biotechnol 2007 7:34.

39. Renilla S, Bernal V, Fuhrer T, Castaño-Cerezo S, Pastor JM, Iborra JL, Sauer U, Cánovas M: Acetate scavenging activity in Escherichia coli: interplay of acetyl-CoA synthetase and the PEP-glyoxylate cycle in chemostat cultures. Appl Microbiol Biotechnol 2012, 95:2109-2124.

40. Castaño-Cerezo S, Pastor JMJ, Renilla S, Bernal V, Iborra JUJ, Cánovas M, Canovas M: An insight into the role of phosphotransacetylase (pta) and the acetate/acetyl-CoA node in Escherichia coli. Microb Cell Fact 2009, 8:54.

41. Valgepea K, Adamberg K, Nahku R, Lahtvee P-J, Arike L, Vilu R: Systems biology approach reveals that overflow metabolism of acetate in Escherichia coli is triggered by carbon catabolite repression of acetylCoA synthetase. BMC Syst Biol 2010, 4:166

42. Cánovas M, Sevilla A, Bernal V, Leal R, Iborra JL: Role of energetic coenzyme pools in the production of L-carnitine by Escherichia coli. Metab Eng 2006, 8:603-618.

43. Cánovas M, García V, Bernal V, Torroglosa T, Iborra JL: Analysis of Escherichia coli cell state by flow cytometry during whole cell catalyzed biotransformation for L-carnitine production. Process Biochem 2007, 42:25-33.

44. Keseler IM, Mackie A, Peralta-Gil M, Santos-Zavaleta A, Gama-Castro S, Bonavides-Martínez C, Fulcher C, Huerta AM, Kothari A, Krummenacker M, 
Latendresse M, Muñiz-Rascado L, Ong Q, Paley S, Schröder I, Shearer AG, Subhraveti P, Travers M, Weerasinghe D, Weiss V, Collado-Vides J, Gunsalus RP, Paulsen I, Karp PD: EcoCyc: fusing model organism databases with systems biology. Nucleic Acids Res 2013, 41(Database issue):D605-D612.

45. Unden G, Bongaerts J: Alternative respiratory pathways of Escherichia coli: energetics and transcriptional regulation in response to electron acceptors. Biochim Biophys Acta 1997, 1320:217-234.

46. Cecchini G, Schröder I, Gunsalus RP, Maklashina E: Succinate dehydrogenase and fumarate reductase from Escherichia coli. Biochim Biophys Acta 2002, 1553:140-157.

47. Cai G, Jin B, Monis $P$, Saint $C$ : Metabolic flux network and analysis of fermentative hydrogen production. Biotechnol Adv 2011, 29:375-387.

48. Murarka A, Clomburg JM, Moran S, Shanks JV, Gonzalez R: Metabolic analysis of wild-type Escherichia coli and a pyruvate dehydrogenase complex (PDHC)-deficient derivative reveals the role of PDHC in the fermentative metabolism of glucose. J Biol Chem 2010, 285:31548-31558.

49. Zhu J, Shimizu K: The effect of pfl gene knockout on the metabolism for optically pure D-lactate production by Escherichia coli. Appl Microbiol Biotechnol 2004, 64:367-375.

50. Hasona A, Kim Y, Healy FG, Ingram LO, Shanmugam KT: Pyruvate formate lyase and acetate kinase are essential for anaerobic growth of Escherichia coli on xylose. J Bacteriol 2004, 186:7593-7600.

51. Baba T, Ara T, Hasegawa M, Takai Y, Okumura Y, Baba M, Datsenko KA, Tomita M, Wanner BL, Mori H: Construction of Escherichia coli K-12 inframe, single-gene knockout mutants: the Keio collection. Mol Syst Biol 2006, 2:2006.0008

52. Cherepanov PP, Wackernagel W: Gene disruption in Escherichia coli: TcR and $\mathrm{KmR}$ cassettes with the option of Flp-catalyzed excision of the antibiotic-resistance determinant. Gene 1995, 158:9-14.

53. Datsenko KA, Wanner BL: One-step inactivation of chromosomal genes in Escherichia coli K-12 using PCR products. Proc Natl Acad Sci USA 2000, 97:6640-6645.

54. Sambrook J, Russell DW: Molecular cloning: a laboratory manual. Thirdth edition. Cold Spring Harbor, New York: Cold Spring Harbor Laboratory Press; 2001.

doi:10.1186/1475-2859-12-56

Cite this article as: Arense et al:: Metabolic engineering for high yielding L(-)-carnitine production in Escherichia coli. Microbial Cell Factories 2013 12:56.

\section{Submit your next manuscript to BioMed Central and take full advantage of:}

- Convenient online submission

- Thorough peer review

- No space constraints or color figure charges

- Immediate publication on acceptance

- Inclusion in PubMed, CAS, Scopus and Google Scholar

- Research which is freely available for redistribution 